\title{
Sur les traces de la post-modernité dans les théories de la communication
}

Richard Godin et Thomas B. Farrell

\section{(2) OpenEdition}

1 Journals

Édition électronique

URL : http://journals.openedition.org/communicationorganisation/1848

DOI : 10.4000/communicationorganisation. 1848

ISSN : $1775-3546$

Éditeur

Presses universitaires de Bordeaux

Édition imprimée

Date de publication : 1 mai 1996

ISSN : 1168-5549

Référence électronique

Richard Godin et Thomas B. Farrell, «Sur les traces de la post-modernité dans les théories de la communication », Communication et organisation [En ligne], 9 | 1996, mis en ligne le 19 décembre 2012, consulté le 19 avril 2019. URL : http://journals.openedition.org/communicationorganisation/1848 ; DOI : 10.4000/communicationorganisation. 1848

Ce document a été généré automatiquement le 19 avril 2019

(c) Presses universitaires de Bordeaux 


\title{
Sur les traces de la post-modernité dans les théories de la communication
}

\author{
Richard Godin et Thomas B. Farrell
}

\section{NOTE DE L'ÉDITEUR}

Traduction par Richard Godin et Thomas B. Farrell

\section{NOTE DE L'AUTEUR}

Titre original : Feature of Life : Traces of Post-Modernity in communication theory.

1 Peut-être s'agit-il d'un signe d'évolution dans l'étude des communications de constater qu'une spécialisation de plus en plus poussée s'accompagne en même temps de réflexions plus audacieuses. Même si les approches esthétique, historique ou philosophique du champ de la pratique des communications n'ont pas livré de preuves empiriques comme le démontre la science "normale", on reconnaît toutefois que ces approches non scientifiques démontrent également des valeurs de rigueur, de profondeur et d'heuristique qui peuvent profiter à l'ensemble de la discipline.

2 Voici quelques explications permettant de clarifier les intentions de ce texte. Premièrement, je démontrerai que la communication humaine est à ce point sousdéveloppée qu'elle requiert une réflexion engagée des penseurs en communication, peu importe leur champ de recherche. Mais cela ne signifie pas pour autant que la théorie scientifique doive être détrônée par une approche purement esthétique ou politique pas plus que tout rejet de la communication puisse être considéré comme une critique non scientifique. Le prix d'une bonne entente entre disciplines est de reconnaître que les éléments d'adéquation, quoique saisissables à travers la diachronie, sont, pour le 
moment, incommensurables. Deuxièmement, les catégories que j'utiliserai pour l'interprétation sont conçues afin de différencier différents parmi niveaux d'analyse dans l'étude dite non scientifique de la communication. Il ne s'agit pas d'une analyse exhaustive : il n'y a pas de doute que plusieurs approches non-scientifiques dépassent le champ de mes compétences. Troisièmement, je ne suis pas un scientifique : ce qui veut dire que la portée de ces réflexions sur la recherche scientifique doit être perçue comme venant des intuitions d'un profane. Je canaliserai mon attention sur les études de base qui représentent une certaine perspective ou qui ouvrent de nouveaux horizons en espérant qu'elles susciteront le désir de prendre connaissance des autres études tout aussi valables que nous n'avons pu aborder.

$3 \mathrm{Au}$ moins trois grands groupes de recherches types offrent d'intéressantes perspectives nouvelles pour l'avancement du savoir de la communication en sciences sociales. Le premier est relatif aux approches philosophiques, lesquelles fonderaient ou étendraient la compréhension de certaines particularités critiques de la communication dans le cadre d'écoles de pensée identifiables. Le deuxième est liée aux approches esthétiquesallégoriques (aesthetic-allegorical). Ici, nous sommes dans le courant le plus actuel de la recherche en communication : comment fonctionne le processus de communication? Les suppositions au sujet de la structure, de l'explication et de la performance sont guidées par les traditions du bon goût et du jugement. La théorie dans ce cadre en est un d'engagement, autant pour les scientifiques que pour les esthétiques. Finalement, se retrouvent les approches interprétatives et critiques, approches par lesquelles l'expérience de la communication, comme le discours et le texte, tend à être formée, recréée et qualifiée en fonction de certaines normes inventées ou dérivées. Il existe une impressionnante littérature à ce sujet mais dont la qualité varie grandement. Dans l'ensemble, ce texte s'attardera aux travaux de recherche liés à la première catégorie (approches philosophiques), tout en faisant des recoupements en profondeur avec d'autres niveaux d'analyse.

4 Comment un tel travail peut-il être évalué à son mieux ? À la lumière de ce qui a été avancé plus tôt dans ce texte, il m'apparaît important d'évacuer l'idée préconçue qu'il ne peut être évalué. Quand vient le temps des études savantes, nous invoquons tout le temps les standards non-empiriques de gradation. Quand un programme de recherche est confronté à la question « Pourquoi ? ", plus d'une fois il faut retourner à la table à dessin. Parmi les standards que nous souhaiterions considérer, nous retrouvons les suivants :

5 1) Consistance analytique : la recherche dérive-t-elle d'une position théorique formulée de manière consistante?

6 2) Rigueur méthodologique: la méthode de recherche est-elle formulée de manière précise, et est-elle appliquée soigneusement? Dans l'éventualité que d'autres chercheurs emploient la méthode, obtiendront-ils approximativement les mêmes résultats?

7 3) Crédibilité basée sur l'intuition: ce standard soulève la pertinence de la recherche humaine dans le courant actuel des pratiques communicationnelles. L'enjeu est de savoir s'il y a suffisamment de divergences entre la recherche et la pratique pour justifier une révision des formulations de recherche;

8 4) Valeur heuristique: la généralisation des résultats de recherche pour les autres chercheurs et différents intervenants. Ce n'est habituellement pas le but visé par la recherche humaniste en communication que de formuler des lois générales et des 
principes universels. Toutefois, certaines recherches devraient à tout le moins donner un aperçu de l'objet d'étude. Mais la meilleure réponse à ce chapitre doit provenir des autres.

\section{Conceptualisation non-scientifique en communication}

9 Il y a une étape en recherche expérimentale au cours de laquelle le chercheur est tenu de rendre opérationnelle sa problématique, par exemple par la mise à l'épreuve à l'aide d'un test préalable. Nous savons tous que lorsqu'une problématique est réduite à ses composantes empiriques, d'autres caractéristiques non-empiriques de la définition sont mises à l'écart. Il devient difficile de déterminer si le résultat, dans chaque cas, est une amélioration (le triomphe de la rigueur sur la pensée approximative) ou une corruption (les idées remplacées par des choses). Afin de connaître les gains et pertes dans la quête des fondements empiriques, il faut s'en remettre à une justification d'une large construction non-empirique de la communication qui exige du chercheur une attention particulière du processus de définition.

10 Le point central de mon approche consiste en l'idée que la communication humaine est silmutanément un processus et une pratique. C'est, comme le dit le vieil adage, la transformation de la matière en énergie avec une signification symbolique. Il s'agit de quelque chose qui se produit involontairement, et quelque chose que nous faisons avec malice et prudence préméditées. Ce double aspect de la communication devient apparent chaque fois que nous nous remémorons des gestes que nous posons habituellement en public ou lors d'une conversation (jouer avec son nœud de cravate, utiliser des expressions bien particulières). Quand ces faits sont portés à notre attention, nous réagissons habituellement avec embarras et agacement. C'est que nous devrions être en mesure de contrôler ce genre de conduite, mais en vain. Dès lors, nous sommes aux aguets, cherchant à anticiper et à épier chacune de nos pensées afin d'éliminer cette mauvaise habitude. Dans cet exemple, la communication n'a jamais abandonné son statut de processus; mais par le phénomène de la réflexion, nous sommes en mesure de nous préoccuper des aspects du processus de communication comme d'une forme de conduite.

11 Dans la perspective que je viens d'esquisser, cela ne nous avancerait à rien de ne considérer qu'un seul côté de la médaille sans s'attarder à l'autre côté. Pour pousser plus loin l'intérêt porté sous toutes les formes d'enquête, dans la mesure où la communication est concernée, il y aura toujours une certaine tension entre la méthodologie employée et l'objet d'études comme tel. Comme la communication est quelque chose que chacun de nous, dans la vie courante, pratique, nous pouvons dire que nous sommes toujours simultanément acteurs et spectateurs. Si certains aspects de notre comportement communicationnel sont ouverts aux explications que peuvent fournir un cadre de contrôle comme les lois, il est possible d'acquérir un ordre élevé dans l'habileté de communiquer seulement via une maitrise des règles et compétences pratiques. Certains modèles de communication interpersonnelle et même culturelle peuvent ultimement être réduits à un construit propre à la communication intrapersonnelle, voire à ses composantes physiologiques. Il appert également que l'intuition est une dimension du savoir communicationnel - une dimension qui peut mettre en contexte l'idiosyncrasie de la plupart des traits de comportement, ce qui pourrait être plus grand que la somme de ses parties. Or, la communication est vue comme un processus si elle répond aux standards d'adéquation de généralisation d'explication dans la communauté scientifique. Ou, elle 
est vue comme une pratique si elle pose également de pertinentes questions à propos de la fidélité, de la véracité et de la qualité de ce que nous disons.

Dès lors que nous sommes toujours acteurs et spectateurs en même temps, il y aura toujours un domaine de la réflexion de notre propre communication pratique qui aura en principe - échappé à notre capacité d'explication. Ce domaine d'explication concerne notre propre performance de communication comme scientifique et chercheur. Voilà ce qu'ont abordé Weimer (1977) et Campbell (1975) lors d'une analyse des caractéristiques rhétoriques de l'entreprise scientifique. D'autres versions plus radicales de la même approche (Cohen, 1972 ; Gouldner, 1973; Weimer, 1983) montrent que les recherches scientifiques de toutes sortes (science pure, science sociale, etc.) impliquent une subjectivité d'un langage limité à établir les fondements et les principes, à sélectionner les objets de recherche, et ainsi de suite. Toutefois, quand les scientifiques sont confrontés à ces allégations, ils répondent habituellement par un «oui, c'est vrai... et maintenant, de quoi parlions-nous justement?»

Je sympathise avec de genre de réponse. Tant que cela peut aider à nous souvenir que nous sommes, après tout, en train de choisir et de regarder les choses d'une certaine manière quand on fait des sciences (ou des arts le cas échéant), cette révélation est loin d'être concluante au point de détrôner l'entreprise scientifique dans ses fondements. Ce qui ressort de tout cela, c'est que la science est elle-même une pratique qui trouve souvent une façon commode de considérer les choses dans un angle donné, de mettre entre parenthèses les aspects pratiques des sujets abordés, en bref, de traiter la communication comme un processus. Dans cette optique, le paradoxe de la science de la communication réside dans l'emploi de moyens techniques et intéressés pour l'étude de sujets sociaux. Par conséquent, la science de la communication est une façon d'enquêter à laquelle le chercheur pourra toujours échapper. Rigueur et régularité de l'explication, le « point de vue de nulle part » décrit par Nagel (1986), sont toujours obtenues moyennant le prix : ici nous sommes dans la réalité, faisant quelque chose quelque part pendant ce temps. Nous avons besoin d'être rappelé sà ce fait à chaque instant, mais une fois cela accompli, il n'y a plus grand chose à dire. Ainsi, je n'ai plus à me porter à la défense des contributions de la science pour une meilleure compréhension de notre part, de la communication. Une fois que nous avons réalisé que la communication comportera toujours des aspects permettant d'élucider la grande élucidation scientifique, peut-être que les engagements momentanés, pourront être mis de côté au profit de notre question centrale : comment le paradigme de la recherche non-scientifique peut-il contribuer à enrichir et approfondir la vue d'ensemble de la communication?

\section{Les approches principales}

La première catégorie de recherches qui sont utiles à ma propre vue d'ensemble comprend ce que je pourrais qualifier d'approches philosophiques. Depuis que la philosophie occidentale a fixé les grandes lignes de ses origines dans le sens du "dialogue ", l'investigation philosophique de la communication ferait ressortir un lien naturel entre méthode et objet de recherche. Cependant, par les temps qui courent, le terme " philosophie » n'indique pas plus clairement le sens commun des hommes qu'il ne fait partie de la nomenclature de notre propre discipline. Non seulement le fait de savoir ce qu'est au juste la philosophie est-il l'un des paradoxes cognitifs les plus discutés, mais il y a aussi une tendance des philosophes eux-mêmes, ce qui provoque leur hilarilité, à 
annoncer la fin de la philosophie. Ainsi de nos jours, la langue de discussion fait davantage avancer les autres branches des sciences humaines. Afin de s'attendre que de telles approches enrichissent notre sujet de discussion, nous devons aller au-delà de ce qui se fait et se dit actuellement sur le sujet.

Les terrains les plus propices à notre vue d'ensemble sont l'herméneutique et la phénoménologie, la sémiologige et le structuralisme, le marxisme et la théorie sociale, et la philosophie non-rationnelle (ou deconstruction). Brusquement présentées, ces approches permettent d'établir certains fondements et principes (ou postulats) a priori à propos de la communication, et ainsi passer directement à un ou plusieurs niveaux d'analyse déjà vus. Le niveau d'analyse (qu'il soit intra, inter, réseau ou structure de système) est alors interprété ou lu presque entièrement à l'aide de postulats. Par la pratique de l'inférence, la philosophie de la communication est en mesure de paraitre à ses partisans suffisament consistante, mais plus ou moins inintellegible non seulement aux yeux des scientifiques de la communication, mais aussi des autres philosophies de la communication. Toutes proportions gardées, voilà ce qu'ils font. Ce qu'ils disent c'est autre chose.

\section{Herméneutique et Phénoménologie}

16 L'herméneutique et la phénoménologie sont différentes du point de vue technique. Mais elles offrent toutes les deux une méthode d'interprétation conçue pour acquérir des connaissances à partir d'un objet de recherche. Toutefois, à la suite de ce simple début décevant survient une foule de développement et de doctrines pour chacune des méthodes de base : du classique au religieux, en passant par l'ontologique et la critique herméneutique; de l'empirique au transcendental, en passant par la phénoménologie heideggerienne (Heideggerian phenomenology) et tout ce qui vient après. Il faut noter premièrement que l'herméneutique et la phénoménologie sont rattachées à certains aspects de la communication à titre d'objet primaire ou d'illustration de leur propre quête philosophique. Deuxièmement, bien qu'elles ne peuvent pas toujours augmenter la valeur du prix de la connaissance, chacune des deux branches de la recherche a quelque chose d'important à livrer à l'étude scientifique de la communication.

Un essai sur la perception de Sloan (1971) trace les origines du concept d'herméneutique à partir des études savantes de la Bible jusqu'à celle de l'interprétation de la littérature. Depuis la parution de cet essai, des chercheurs dans une multitude de disciplines ont trouvé dans l'herméneutique un outil d'analyse utile pour la compréhension des caractéristiques de la communication négligées jusqu'alors. Spécifiquement, l'herméneutique porte à notre attention le problème de sens posé par la présence de deux acteurs ou plus lorqu'ils doivent abandonner leut subjectivité dans le cadre d'une rencontre avec le monde des «autres ». Le monumental ouvrage de Gadamer, Truth and Method (1975) touche à cette ligne d'influence en appliquant la métaphore de la conversation à l'interprétation de la littérature. Les corrélations qu'il tire sont cependant pertinentes pour les étudiants qui s'intéressent à l'art négligé de la conversation. L'une des observations que Gadamer fait au sujet de la conversation voudrait que nous soyons moins le meneur que le mené, et que la conversation n'est pas quelque chose de planifié mais plutôt quelque chose dans lequel nous tombons (fall into). Ce que Gadamer élabore après tout, c'est une théorie de l'interprétation critique qui elle n'est pas un raccourci par l'utilisation du jugement comme instrument arbitraire. Le paradigme de la conversation 
de Gadamer est d'autant plus intéressant pour les scientifiques de la communication, qu'il vaut la peine que l'on s'y arrête en raison des trois considérations suivantes :

1) Sa notion de texte est communicative dans le sens qu'elle permet d'enrichir notre conception de l'analyse du message ;

2) Il admet la possibilité de la théorie de l'agent central en communcation, sans présupposer que chaque étape de la communication soit contrôlée stratégiquement ;

3) Il n'impose pas de règles édictant le contexte des noyaux de sens (contextual layer of meaning) comme une dimension possible de la compréhension (Gadamer, 1976).

Cela a déjà été dit, mais, l'intention et la méthode de Gadamer sont manifestement étrangères aux préceptes et contrôles de prédiction traditionnellement appliqués dans les sciences sociales. De même, ce formidable penseur contemporain a une manière déconcertante de présenter les théoriciens de la connaissance sociale qui envisagent la voix passive comme processus de contribution active. Nous «sommes menés " signifie "parle lui-même par nous", et ainsi de suite (So we "are led", meaning "speaks itself through us", and so forth.). Ultimement, Gadamer commence avec certains principes fondamentaux de la communication et il est mené (is led) à destination en ayant toujours à l'esprit ce qui suit: l'esthétique contemplative est un refuge réconfortant. Pour les rhétoriciens, l'ouvrage de Gadamer est trop rapidement parcouru. Ce que l'auteur trouve réconfortant dans la mise en place des interactions, est insuffisant et inapplicable pour d'autres. Pour toutes les difficultés d'application, ou peut-être à cause d'elles, le travail de Gadamer se distingue par ses affirmations quant à la communication intrapersonnelle et les domaines de la communication publique, affirmations qui vont à contre-courant.

Schutz (1964) apporte aux pratiques du monde de la communication un mariage plus pragmatique de l'herméneutique interprétative et de la phénoménologie. Dans une vaste série d'études utilisant la littérature classique, la philosophie pragmatique et la recherche sociale, Schutz tente de faire une transition du domaine de l'interprétation individuelle vers l'interpersonnelle et les aspects sociaux de la connaissance. Pendant que nombre d'études de Schutz semblent avoir été conçues en regard des types sociaux -l'étranger, le citoyen bien informé, le revenant (?)- (social types) (the "stranger", the "well-informed citizen", the "homecomer»), ces types se combinent de manière récurrente à des problèmes de communication. D'ailleurs, Schutz donne à ses sujets une richesse d'observations significatives qui peuvent être modélisées en termes de développement relationnel, de résaux d'amis et de communication politique.

Une foule de chercheurs contemporains revendiquent de nouveau l'importance de l'herméneutique et de la phénoménologie pour la bonne compréhension des pratiques de la communication : l'ouvrage de Apel: «The A'Priori of Communication» (1972); celui de Edie : Speaking and Meaning (1976) ; les études d'interprétation, de textualité, de temps et d'action de Ricœur $(1974,1976,1977,1984,1985)$, et finalement Schrag avec Communicative Praxis and the Space of Subjectivity (1985). Ces ouvrages soulèvent de fascinantes questions à propos du sens et de la compréhension des pratiques de la communication. Il y a bien sûr un biais compréhensible à travers toute cette littérature envers la subjectivité intrapersonnelle du domaine de la communication. Malgré le fait que nombre de constructions théoriques et de variables dans l'étude scientifique de la communication aient dérivé de ce même royaume intrapersonnel, cela ne devrait pas réduire pour autant l'inestimable valeur ajoutée provenant de la recherche en communication. 
21 La plupart des meilleurs chercheurs emploient leur propre test heuristique découlant des approches herméneutique et phénoménologique. Hyde et Smith dans «Hermeneutics and Rhetric: A Seen But Unobserved Relationship" (1979) soulignent l'influence de la phénoménologie heideggerienne dans la problématique entourant la création et la découverte de sens dans les textes de communication. Les travaux de Hyde, une étude portant sur l'anxiété (1980) et une autre sur les problèmes liés aux technologies de communication (1982), donnent avec force un exemple de l'utilisation de la valeur heuristique en tentant de savoir comment une méthode philosophique peut enrichir l'élaboration de définitions en science de la communication. Le travail de Stewart (1981) portant sur le dialogue logique (dialogic) en communication doit énormément à une variété d'influences phénoménologiques, et en cela tente de manœuvrer dans le but de rendre le tout compatible avec l'ensemble des pratiques existantes en sciences sociales. Les programmes extensifs de recherche sur le constructivisme ont été influencés par plusieurs générations de disciples de la ménoménologie chutzienne. Cette recherche liée aux fondements phénoménologiques poursuit son cours en étudiant les constructions discursives sur la base d'une mesure empirique d'un champ pré-sentationnel. Ainsi, il n'existe pas d'évidence concernant les enquêtes approfondies sur le champ des constructions discursives, ce qui procure au constructivisme un avenir qui semble assuré. La revue Western journal of Speech Communication (1986) consacrait un numéro entier à ce sujet (" Communication and Consciousness »).

22 Ce survol de l'herméneutique et de la phénoménologie montre que la recherche en cette matière se concentre sur l'acteur pour mieux comprendre la communication, tout en offrant par ailleurs une méthode féconde pour la construction de définitions et leur développement. Comme le notent les spécialistes en herméneutique, les chercheurs en communication, comme tous ceux qui utilisent les communications, sont constamment confrontés au phénomène de la pluritextualité (multitextual phenomenon).

Nous sommes immergés, tout au long de nos existences, tant sur le plan privé que public, dans la communication à des niveaux divers, soit intrapersonnelle, interpersonnelle, organisationnelle et systémique. Heureusement, ni le chercheur ni le praticien en communication ne sont capables de prêter attention à tous les niveaux d'interprétation au même moment. Toutefois, à la lumière des propos tenus par mes collègues, nous commencerions à nous questionner sur les différents niveaux et les influences prioritaires de chacune. Or, l'herméneutique et la phénoménologie seraient parmi les meilleurs outils pour arriver à comprendre les enjeux qui y sont liés.

\section{Sémiologie et Structuralisme}

24 La sémiologie et le structuralisme sont suffisament près, conceptuellement, l'une de l'autre pour pouvoir tirer profit de leurs différences. Néanmoins, j'aimerais souligner certains points communs dans les thèmes et ainsi mettre l'accent sur certains ouvrages qui ouvrent la porte à une nomenclature. La sémiologie présente au point de départ une difficulté en ce sens qu'elle se définit d'une certaine manière comme une sorte de science. Je ne l'évaluerai pas sur ce point, mais j'attirerai simplement votre attention sur ce qui la caractérise.

Les différents liens qui unissent la sémiologie prennent source à deux grands fondements conceptuels. Le premier étant lié à l'important travail du philosophe et logicien américain 
Charles Sanders Peirce. Sa théorie des signes était issue de la phénoménologie de la perception et du sens qui s'appuie en partie sur la source du savoir contemporain, soit le modèle linéaire de la communication information-transmission tronqué quelque peu par la lorgnette culturelle de la linguistique française. Cela expliquerait pourquoi Barthes (1968) a élaboré à partir de sa critique hautement impressionniste de la sémiologie un modèle mathématique de codes et de transformation. Le résultat offrant en une extravagante diversité, nous sommes chanceux de pouvoir compter sur plusieurs ouvrages utiles d'introduction.

Les origines philosophiques de la sémiologie ont été colligées par Lyne (1980). Spécifiquement centré sur l'influence sémiologique de Peirce, l'ouvrage de Lyne nous introduit aux premières interprétations systématiques des signes et des fonctions en découlant, expliquant comment les postulats philosophiques logiques de Peirce conduisent en un développement logique des signes.

Parmi les ouvrages d'ensemble les plus accessibles portant sur la sémiologie contemporaine, notons celui de Leach, Culture and Communication (1976). Bien qu'écrit pour un public initié mais non spécialiste, ce livre d'une grande simplicité répond tout de même aux préoccupations des chercheurs en communication. Ce qui ressort de cet ouvrage est son traitement didactique soigné. Les termes de bases sont définis. Nous y lisons comment le rapport entre signes et symboles contribue à la création et à la découverte d'une riche diversité de contextes. Le plus important, nous apprenons comment la sémiologie peut être employée comme méthodologie de recherche en communication. La conceptualisation de la communication se fonde sur l'alliage sensévénement qui est constitué par les signes et leur interprétation. Cette perspective contredira nombre de postulats des grands courants de recherche, mais Leach se fait prudent en offrant une liste détaillée d'auteurs classiques (Malinowski, Lévi-Strauss, Jakobson).

La tradition française en sémiologie nécessite une série d'introductions. Je recommande Sémiologie (1975) de Guiraud et Éléments de sémiologie de Barthe. Ces derniers examinent virtuellement un territoire conceptuel identique : signes, signifiés et systèmes, codes et variations, dénotation et connotation, et programmes pour des recherches relatives à la sémiologie. Les deux présument que la sémiologie est une science de la communication soigneusement délimitée de l'extérieur par un cadre de référence.

L'étude de la sémiologie a pris un virage intéressant lorsqu'abordée par des chercheurs issus du récent développement de l'anthropologie sociale. Parmi ceux-là, Geertz et Douglas partagent avec les sémiologues la conviction que la communication est dépendante d'un cadre de référence interne lié à une culture commune. Différents des sémiologues, ces chercheurs sont davantage préoccupés par la symbolique que par les signes. Ils croient que le sens commun de la logique fonctionne à l'intérieur des limites de la communauté culturelle. En raison de cette croyance, ils admettent que le temps change, voire transforme les systèmes de symboles. Geertz (1983) présente une démarche provocante insistant sur les limites du discours dans les systèmes de connaissance, qui doit subir une révision, tout comme l'ensemble de notre discipline. Douglas reste plus près du moule de la sémiologie, largement parce qu'elle refuse implicitement la dimension actionnelle dans toutes les logiques au cours de ses découvertes (Douglas, 1954, 1960,1982). Traçant une nouvelle voie, elle a tendance à traiter les symboles comme s'il s'agissait de signes. 
30 La littérature en sémiologie et en anthropologie sociale est beaucoup plus volumineuse que ce qui est décrit plus haut. Mais quelques conclusions partielles seraient appropriées ici. A première vue, il appert qu'un vaste territoire de recherche reste encore inexploré pour la recherche en sciences sociales. Bien que les postulats qui guident les sémiologues semblent diamétralement opposés à ceux rencontrés en savoir social, il existe d'excitantes perspectives de développement dans la recherche sur les réseaux relationnels et généralement les variables de messages, sans parler de l'étendue des zones grises concernant l'étude des médias où l'analyse de contenu fait le lien avec la recherche critique. Cela dit, de bonnes raisons expliquent toutefois pourquoi si peu de travail a été accompli sur ce chapitre. Les vocabulaires des sémiologues et anthropologues sont imposés, et il y a une tendance dans chaque école de pensée à traiter l'objet de recherche comme une matrice relationnelle dans laquelle la rigueur est entièrement intégrée. Bien qu'il n'existe pas de loi sémiologique contre la recherche expérimentale, de manière unanime, sa logique se dresse comme telle à l'extérieur des présomptions probabilistes de l'expérimenation des sciences sociales.

31 L'anthropologie sociale est dans une situation quelque peu différente, et pourrait offrir un vigoureux correctif à la tendance psycho-réductionniste dans la communication intrapersonnelle. Déjà, la variable culturelle est d'une rare commodité dans la recherche en communication (Katriel et Philipsen, 1981, constituent un exemple « rafraîchissant »). À la sémiologie et à l'anthropologie sociale, le message est une métaphore linguistique vague pour un large système socioculturel relationnel. Toutefois, pour nombre de chercheurs en communication, le large système relationnel est une métaphore relationnelle vague quel que soit le message. Cette inversion conceptuelle n'est pas à nier, car certaines discussions entre systèmes prouveront un jour son utilité.

\section{Marxisme et théorie sociale}

Les grands affrontements contemporains que se sont livrés humanistes et scientifiques peuvent être trouvés à travers le marxisme et la théorie sociale. Nous avons noté la tendance des sciences sociales à mettre entre parenthèses ou ignorer les conditons ou/et les circonstances extérieures de son objet dans la définition et le modèle d'explication de la communication. L'une des raisons de cette confusion entre théorie sociale et sciences sociales (sans compter le jargon germanique), c'est cette tendance qui consiste à retirer les parenthèses dans la recherche en sciences sociales pour prendre en compte que ce que l'on ignorait jusqu'alors pour prétendre à la science. La source de cette ambiguïté peut être retracée à l'origine des textes de la théorie sociale : aux travaux de Marx lui-même.

Dans une laborieuse mais puissante analyse du marxisme, Gouldner (1980) propose la présence d'une ambivalence fondamentale dans le marxisme entre la nécessité comme déterminisme (c'est-à-dire que les conditions matérielles ont un rapport causal avec sens et action) et la nécessité comme impératif (la signification rhétorique qui doit être actée). Cette ambivalence renseigne toute chose, à partir de la formation consciente dans l'action révolutionnaire, jusqu'au statut des produits culturels dans l'ordre social. D'ailleurs, l'ambivalence est englobée par des concepts contraignants mais incertains comme la " totalité », la "superstructure ", même l'idéologie. Comme résultat de ce problème initial, nous retrouvons ce qui peut-être qualifié de marxisme scientifique aussi bien que de marxisme révolutionnaire romantique. Le marxisme scientifique se préoccupe de la formation des conditions qui mènent à une situation révolutionnaire. Le marxisme 
révolutionnaire romantique, croyant que la pensée, le désir et la volonté sont les ingrédients de transformation de la société, est considérablement moins stable. Mal à l'aise avec la science en général, il donne dans l'exhortation utopique (la révolution à tout prix), et de manière prédictible, prolonge le pessimisme et la mélancolie. Les deux se fondent sur les textes originaux de Marx, et chacun trouve des adhérents contemporains. Le groupe de penseurs de l'École de Francfort est le plus manifeste des exemples d'utopie de marxisme romantique et de théorie sociale. C'est de là qu'émerge la critique la plus virulente à l'égard du positivisme en sciences sociales. Horkeimer, Adorno, Bloch, Benjamin et Marcuse sont, à l'origine, des critiques sociaux possédant un héritage religieux. Comme je le soutenais à un autre moment (Farrell et Aune, 1979), la position critique de l'École de Francfort était si catégorique qu'elle excluait virtuellement tout discours pratique. Dans un ouvrage poursuivant un thème semblable, The Tragedy of Enlightenment, Connerton (1980) argue que l'École de Francfort s'est elle-même bâillonnée, paralysant ainsi son action politique. Je passe rapidement sur ces motifs autodestructeurs que Connerton a établis dans l'esprit de la pensée de cette école, mais il s'agit là d'une interprétation provocatrice.

Il y a, parmi les détracteurs du marxisme romantique, plusieurs contributions à l'étude des communications. L'invraisemblable mélange Freud-Marx-pragmatisme du langage primitif conduit au fascinant ouvrage sur la communication dans la famille (l'École de Francfort dirait qu'elle a créé ce champ de recherche), les distorsions de la propagande et généralement la communication administrative. Peut-être un ouvrage capital: La Personnalité autoritaire (Adorno, Frenkel-Brunswick, Levinson et Sanford, 1950). Les modèles de communication généréspar les théories de l'École de Francfort sont simplistes, au point même que des théoriciens contemporains, comme Schiller (1969), emploient un point de vue mimétique de la domination des médias réduisant ainsi toute la communication au statut de la variable dépendante de produit. Pour toutes ces difficultés, il y a un sens par lequel le véritable développement d'une science de la communication est redevable à cet assortiment dépareillé d'intellectuels. L'analogie qui vient à l'esprit est l'origine paradoxale de la sociologie contemporaine de Spencer et Comte.

Il existe aussi un marxisme scientifique et une théorie sociale. Ici, il est difficile de ne pas penser à Habermas, dont la contribution significative à la philosophie, l'éthique, la critique, l'histoire, l'art, la linguistique et la communication défie toutes les limites des disciplines conventionnelles. Depuis les années 50, alors assistant de recherche pour Adorno, Habermas a travaillé sur un vaste projet philosophique conçu pour ravigorer la critique sociale tout en maintenant en place les fondations des sciences naturelles et sociales. Habermas est constant quant à son intérêt pour la communication sociale. Les premières études, Connaissance et intérêt humain (1971) et Théorie et pratique (1974), sont empreintes de conventionnelles questions philosophiques sur l'épistémologie et la connaissance qui plongent leurs racines dans la pensée allemande. Ces ouvrages sont néanmoins importants en raison de leur engagement direct vis-à-vis des méthodes et programmes de recherche en sciences sociales, aussi bien que pour le très discuté virage linguistique de la théorie critique. En revitalisant le plus moderne héritage de l'École de Francfort, Habermas cherche à réinterpréter Marx dans une perspective communicationnelle. En réintroduisant certains postulats de Kant, qui présupposent une pratique véritable d'une réflexion du dialogue logique (une pratique optimum de la communication), Habermas admet sous les aspects de la raison et de la critique que le 
déterminisme historique ne joue pas, et que par conséquent, le poids cummulatif des événements ne peut l'annuler seul. Pour lui, alors, le projet de modernité n'est pas mort et la perspective de la raison est toujours valable. En d'autres mots, il n'y a pas de contradiction entre les intérêts que nous avons dans notre recherche et la validation de ce que révèle cette recherche via la médiation des autres. La validation est fondamentalement commmunicative. La théorie communicationnelle de Habermas incorpore des aperçus de la philosophie analytique, de l'argumentation, de la théorie des actes du discours et de l'analyse des systèmes, colligées avec une dose de pragmatisme venue de l'Ouest. Ce qui résulte en une vision du discours ordinaire centrée sur le langage, à l'auditoire dépendant, fondée sur la réflexion rationnelle et admettant une logique développementale communicative et (plus tard) une compétence morale. Chacun des aspects de cette théorie a reçu une attention particulière de la part des chercheurs. Au-delà du contenu provocateur des concepts de Habermas, s'y retrouvent un engagement partagé sur les méthodes et les enjeux importants dans la recherche en sciences sociales. Pourtant, il n'y avait pas jusqu'alors de programme de recherche empirique systématique pour introduire les aspects pertinents de la théorie elle-même. Aujourd'hui, la bibliographie existante sur Habermas suffirait à elle seule à remplir ces pages. Je recommande vivement l'ouvrage de McCarthy (1978), The Critical Theory of Jurgen Habermas, qui est une introduction complète à l'œuvre. Habermas propose un agenda inventif pour les scientifiques en sciences sociales intéressés par la phase de construitformation de la théorie du développement en communication. Nous proposons, pour varier sur le même thème, deux autres théoriciens qui ont pris des chemins bien différents.

Dans System and Structure: Essays in Communication and Exchange, Wilden défie toutes les catégories de systèmes que je peux imaginer. Ce difficile ouvrage peut être considéré comme un amalgame de connections réprimées chez les philosophies sociopsychologiques et la recherche des paradigmes. La communication est vue comme un modèle de l'échange et un concept fondamental de l'échange d'informations. Mais Wilden (l'un des fondateurs de la tradition marxiste britannique) est au fait des terminologies des ramifications politiques. Chacune est une sorte d'expérience dans les politiques des sciences sociales. D'une analyse de type Lacan du phallocentrisme dans presque toute la littérature thérapeutique post-freudienne, le structuralisme nous livre une analogie aux lois du capitalisme tardif, et ainsi de suite. Les travaux de Wilden produisent un inventaire virtuel de réflexions fascinantes sur les conséquences des modèles des sciences sociales comme communication comportementale (communication behavior).

Une indication de la profondeur de la tradition marxiste britannique peut être cernée dans un ouvrage de Giddens : A Contemporary Critique of Historical Materialism (1981). Ce livre important est plus intéressant en ce qui concerne l'engagement des médias de communication dans les écrits originaux de Marx que sur le tour de force que constituent les variations de la théorie sociale radicale. Le résultat est unique: une théorie de structuration qui n'est pas liée au processus de déterminisme développemental. Du point de vue de Giddens, l'acquisition et la concentration du pouvoir n'est pas entièrement la conséquence d'une production contrôlée du savoir. Le pouvoir équivaut au contrôle de l'information à distance et à la capacité de surveillance et de discipline sur ceux qui dévient des normes de conduite. Giddens argue alors que l'enjeu et les règles entre systèmes conflictuels changent, même lorsque coexistent en parallèle dans un court laps de temps différents types de système développemental. C'est une vue non révolutionnaire 
de l'histoire. Il n'est pas nécessaire d'endosser la théorie critique des agendas comme Giddens pour apprécier dans cette perspective l'analyse sociale et la recherche empirique. L'approche de Giddens et, plus généralement la théorie sociale, représente ce type de défi qui figure dans les meilleures contributions de la philosophie de la communication. Le recherche en communication sociale porte davantage sur le langage et les mots que sur les nombres. Pour mieux dire, les mots et les nombres sont spécifiquement des outils destinés à saisir des données invariables de phénomènes plus évasifs : les rapports de sens entre les personnes.

\section{Philosophie non-rationnelle ou déconstruction}

La présomption selon laquelle l'on peut faire des affirmations sûres et véritables à propos de la vie sociale fait appel à une quatrième catégorie générale de spéculation de la pensée : la philosophie non-rationnelle ou la déconstruction. Bien que je n'aie fait aucune tentative pour saisir les nuances de cet amorphe champ de recherche, j'en discuterai de manière générale et prudente, profitant de l'occasion pour conclure sur l'ensemble de ce travail.

La théorie de Derrida nous permet de parler de mouvements éloignés du rationalisme. Plusieurs ouvrages interprétatifs utiles introduisent le profane à cet imposant philosophe français, incluant Prophets of Extremety (1985) de Megill et The World, the Text, and the Critic (1983) par Said : pour une critique de Derrida seulement, il faut consulter Rorty (1982). Tous ces ouvrages concèdent l'immense défi de déconstruction imposé à une lecture conventionnelle du discours centré sur l'émetteur en communication.

Bien qu'il existe des différences majeures et controversées entre Foucault et Derrida, ce qui les lie c'est ce rejet de ce que l'on appelle la préoccupation «logocentrique » de la pensée occidentale en général prise dans le carcan d'une primauté de la singulière connaissance du sujet dans les textes de la philosophie classique (Platon,

Aristote, Descartes, Kant, Hegel). Si la philosophie est morte, si elle n'a pas satisfait sa quête de la vérité qui doit être connue pour son unicité, une des raisons possibles est que la quête était terriblement à l'envers depuis le début. L'idée réelle d'un rapport stable sujet-objet à la vérité est sans fondement. Pour Derrida en particulier, ce logocentrisme stable est caractéristique dans la primauté et il croit que le discours se fond à l'histoire des idées. Avec le discours, nous nous assurons un émetteur qui pense ce qu'il dit, un récepteur à qui le sens apparaît, et ainsi de suite. Notez que cette condition particulière de vérité communicationnelle se rapproche de celle de Habermas. Mais, le filet de la philosophie non-rationnelle attrape une plus grande quantité de nemesis que la théorie sociale et la philosophie analytique. L'idée est de sortir le sujet du discours, de remplacer la primauté du discours par le phénomène de l'écriture ou le texte, et de remplacer la quête de la vérité par l'interprétation du sens (ou mauvaise interprétation ou misreading). Quand Rorty (1982) dit que la littérature critique est la nouvelle philosophie, voilà ce qu'il pense (ou du moins c'est l'une des choses que son texte signifie). Dans l'ensemble, le processus de mauvaise interprétation systématique vise à connaître les intentions de l'auteur davantage que de savoir ce que le texte avance en confrontant l'auteur à son savoir. C'est ce que ces chercheurs réfèrent à la déconstruction.

43 À ce point-ci, les visées de Derrida et Foucault prennent des directions précises bien différentes. Foucault, le plus engagé des deux politiquement, cherche à savoir dans quelle 
mesure les règles de formation du discours détermine ou exclut la participation appliquée à diverses formes d'affiliation. Les définitions de raison et folie, de culpabilité et responsabilité, de discipline et autorité, de genre et autonomie offrent des marges inexplorées de pouvoir et de domination. Encore là, ce n'est pas tant le fait que le langage soit contrôlé par un sujet que le fait que le langage lui-même contrôle son usage. Pour Foucault, la déconstruction est une tentative concertée afin de voir ce qui a été exclu du cadre. Pour Derrida, en dépit de ses protestations, la pratique est plus académique. En réinventant le sens à donner à nos textes classiques, il espère restaurer quelque chose de la fluidité et de la richesse dans la perspective de la signification dans le langage.

Pour les théoriciens de la communication, j'estime qu'il est plus intéressant de lire les ouvrages portant sur les ouvrages de la déconstruction, que les ouvrages proprement dit. Foucault $(1972,1973,1977)$ et Derrida $(1972,1974,1981)$ ont à peu de choses près la même influence sur les sciences humaines que Khun et Popper ont dans une vision progressive de la science. Ils représentent le contentieux des penseurs qui dérangent l'ordre établi, qui aiment voir la pièce trembler et les gens avoir peur. Pourquoi alors l'étudiant en communication devrait -il prendre au sérieux ce genre de vandalisme cognitif? Parce qu'ils ont exploité une situation mythique à propos de la façon de faire des communications et ont pris leur faits d'armes pour un engagement extrêmement dérangeant.

\section{Conclusion}

La communication chez les personnes semble être plus en lien avec l'invention de la connaissance qu'avec la recherche de la vérité. Ajoutez à cela le truisme voulant que l'agent de communication ne soit jamais capable d'exercer un contrôle total sur les symboles, les signaux, les expressions, les unités de message et le sens de l'information. Néanmoins, comme théoriciens, nous pouvons prétendre pouvoir faire non seulement du sens, mais aussi de réelles affirmations concernant les phénomènes de communication en général et d'en expliquer les caractéristiques. Vu de la perspective de Foucault et Derrida, le processus entier d'expérimentation scientifique a plus en commun avec la déconstruction que nous le supposons. Je ne dis pas cela pour discréditer leur approche, mais pour les remettre en perspective.

La science de la communication progresse au point qu'elle génère des hypothèses qui peuvent être invalidées.

Même si nous évoluons dans un univers de théorie de la décision, de communication de masse, de déception, de cognition interpersonnelle, de réseaux d'analyse, reste que nous recherchons une certaine cohérence qui n'est pas isolée des choix et du hasard du monde environnent, mais pourrait être renversée par leur insinuation. Au mieux, nous obtenons une vérité fragile. Elle est enrichie par les différentes approches proportionnelles du savoir communicationnel offert par les écoles actuelles de pensée. Chaque perspective que j'ai examinée possède son propre sytème catégoriel pour une pratique d'ensemble de la communication. Conçues à partir de ces systèmes, les différentes approches étudient l'argumentation, les règles d'approche, les mythes, les rituels, les textes et métaphores, chacune offrant une invention conceptuelle aussi bien qu'un potentiel du point de vue duquel nous faisons la communication. Il n'y a pas de contradiction à penser en fonction de l'herméneutique, la sémiologie, la théorie sociale ou même la déconstruction et à penser en termes de science de la communication. Il y aura toujours d'extraordinaires 
tensions. Mais c'est par ces tensions extraordinaires qu'émergent des possibilités extraordinaires qui font que la science de la communication persiste. 